\title{
Physical activity and cancer: an umbrella review of the literature including 22 major anatomical sites and 770000 cancer cases
}

\author{
Leandro Fórnias Machado de Rezende, ${ }^{1}$ Thiago Hérick de Sá, ${ }^{2}$ Georgios Markozannes, ${ }^{3}$ \\ Juan Pablo Rey-López, ${ }^{4}$ I-Min Lee, ${ }^{5,6}$ Konstantinos K Tsilidis, ${ }^{3,7}$ \\ John P A loannidis, $8,9,10,11$ José Eluf-Neto ${ }^{1}$
}

\begin{abstract}
- Additional material is published online only. To view please visit the journal online (http://dx.doi.org/10.1136/ bjsports-2017-098391).

For numbered affiliations see end of article.
\end{abstract}

\section{Correspondence to}

Mr Leandro Fórnias Machado de Rezende, Departamento de Medicina Preventiva,

Faculdade de Medicina FMUSP, Universidadede São Paulo, São Paulo, Brasil; lerezende@usp.br

Accepted 31 October 2017 Published Online First 16 November 2017
Check for updates

To cite: Rezende LFM, Sá TH, Markozannes G et al. Br I Sports Med 2018:52:826-833.

\begin{abstract}
Objective To provide an overview of the breadth and validity of claimed associations between physical activity and risk of developing or dying from cancer.

Design Umbrella review.

Data sources We searched Medline, Embase, Cochrane Database and Web of Science.

Eligibility criteria for selecting studies Systematic reviews about physical activity and cancer incidence and cancer mortality in different body sites among general population.

Results We included 19 reviews covering 22 cancer sites, 26 exposure-outcome pairs metaanalyses and 541 original studies. Physical activity was associated with lower risk of seven cancer sites (colon, breast, endometrial, lung, oesophageal, pancreas and meningioma). Only colon (a protective association with recreational physical activity) and breast cancer (a protective association with overall physical activity) were supported by strong evidence and highly suggestive evidence, respectively. Evidence from endometrial, lung, oesophageal, pancreas and meningioma presented hints of uncertainty and bias in the literature $\left(e g\right.$, not reaching $P$ values $\left.<10^{-6}\right)$ showing large between-study heterogeneity and/or not demonstrating a definite direction for the effect when $95 \%$ prediction intervals were considered. Four of the 26 meta-analyses showed small study effects and 4 showed excess significance.

Conclusion Physical activity is associated with a lower risk of several cancers, but only colon and breast cancer associations were supported by strong or highly suggestive evidence, respectively. Evidence from other cancer sites was less consistent, presenting hints of uncertainty and/or bias.
\end{abstract}

\section{INTRODUCTION}

Physical activity has been traditionally linked with lower risks of colon and breast cancer. ${ }^{1-6}$ Annually, thousands of new epidemiological studies are conducted and published to examine whether physical activity may also decrease risk of other types of cancer. Recently, prospective cohort studies ${ }^{78}$ and meta-analyses have claimed that physical activity might be additionally associated with cancer of bladder, ${ }^{9}$ endometrial, ${ }^{10}$ oesophageal, ${ }^{11}$ gastric, ${ }^{12}$ glioma, ${ }^{13}$ kidney, ${ }^{14}$ lung, ${ }^{15}$ meningioma, ${ }^{13}$ ovarian, ${ }^{16}$ pancreas ${ }^{17}$ and prostate. ${ }^{18}$ If these associations are causal, a substantial burden of cancer could be avoided worldwide given the high prevalence of physical inactivity. ${ }^{19}$

Another possibility is that some claimed associations about physical activity and cancer could be explained by biases in the literature. There is strong evidence that studies showing positive and significant results are more likely to be published than studies with negative and non-significant findings. ${ }^{20}$ These sorts of publication bias and outcome reporting bias threat validity of scientific evidence, ${ }^{2122}$ cause general public anxiety and scepticism $^{23}$ and misguide clinical and public health decisions. In fact, bias has been suspected in cancer epidemiology literature about presumed risk and prognostic factors, biomarkers and multiple carcinogens. ${ }^{24-31}$ Nevertheless, neither the international organisations (eg, International Agency for Research on Cancer-IARC; World Cancer Research FundWCRF) ${ }^{2}{ }^{32}$ nor the most comprehensive systematic reviews and meta-analyses on physical activity and cancer have considered the array of analytical procedures available to detect hints of uncertainty and bias in the body of evidence to state their conclusions (see online supplementary table S1).

We performed an umbrella review 2627303133 of systematic reviews and meta-analyses to provide an overview of the breadth and validity of claimed association between physical activity and risk of developing or dying from cancer. We comprehensively evaluated the robustness of evidence between physical activity and cancer, appraising hints of uncertainty and bias in the body of literature.

\section{METHODS}

\section{Literature search}

We searched Medline, Embase, Cochrane Database of systematic reviews and Web of Science for systematic reviews published up to 22 November 2016 , aiming to investigate the association between physical activity and risk of cancer incidence and cancer mortality. Online supplementary table S2 in the appendix shows the search strategy. We also reviewed the references list of the eligible reviews.

\section{Selection of reviews and methodological quality assessment}

We only selected systematic reviews (irrespective of performing meta-analyses) evaluating the association between physical activity and cancer among the general (healthy) population. Whenever more than 
one eligible systematic review addressed the association between physical activity and the same cancer site, we selected the review with the largest number of studies included. We selected more than one review per cancer site whenever the reviews with the largest number of individual studies were restricted by study design or cancer subsite. In these cases (ie, breast, colon and rectum cancers), data analyses were performed separated by review to avoid overlap of studies. We excluded systematic reviews of prognostic studies, reviews that did not systematically search the literature and reviews that did not provided comprehensive data from individual studies (specifically information listed in the data extraction section). Two researchers (LFMR and JPRL) independently selected the eligible reviews after screening consequently titles, abstracts and full texts. A third researcher (THS) settled disagreements between authors. The list of included and excluded reviews is available in the online supplementary table $\mathrm{S} 3$ in the appendix.

We assessed the methodological quality of the included reviews using the AMSTAR too ${ }^{34}$ and gathering data on statistical analyses used to assess hints of uncertainty and bias in the body of evidence.

\section{Data extraction}

We extracted author's name, year of publication, number of studies included (by study design), physical activity domains (eg, recreational, occupational, total), and maximally adjusted summary estimates from the systematic reviews. For each individual study in a systematic review, we extracted authors, year of publication, study design (case-control or cohort), sex, physical activity domain, period in life which of physical activity was measured, number of cases and controls (for case-control studies), number of cases and sample size (for cohort studies), cancer indicator (incidence, mortality or incidence and mortality), maximally adjusted measure of association and its respective 95\% CI comparing high versus low categories of physical activity. Data extraction was independently performed by two researchers (LFMR and JPRL), with discrepancies solved by a third researcher (THS).

\section{Statistical analysis}

Primary analysis

The primary analysis in this umbrella review focused on two approaches to evaluate the association between physical activity and cancer: (1) 'Any physical activity': if individual study presented multiple measures of association by physical activity domains, we selected one based on the following order: total physical activity, multiple physical activity domains, recreational, commuting, occupational and household physical activity. We considered 'total physical activity' any estimate with all four physical activity domains and 'multiple physical activity' an estimate with a combination of at least two, but not all, domains. The 'any physical activity' approach was the most comprehensive analysis since it included the largest number of estimates per meta-analysis; (2) Recreational physical activity: we performed data analysis using all individual studies presenting recreational physical activity estimates. Recreational physical activity is the most studied and the more easily modifiable physical activity in comparison to other domains.

Both approaches used in the primary analysis included only cohort studies (ie, case-control results were considered in the stratified and subgroup analyses) and one measure of association per individual study. Whenever a measure of association was not available for the total sample in an individual study (eg, men and women relative risks (RRs) estimates were provided separately), we performed fixed effect models to estimate summary effects between categories and included the latter in the meta-analysis. In addition, one systematic review ${ }^{17}$ presented multiple estimates based on timing in life measures for the same physical activity domain (eg, distant past, recent, consistent over time) and we selected the consistent over time measure (same criteria used by the authors). ${ }^{17}$

\section{Stratified and subgroup analyses}

We also performed stratified analyses combining estimates by study design (case-control and all study design-cohort and case-control), sex and other physical activity domains (eg, total physical activity, occupational physical activity) within and across studies. In addition, considering all study designs, we reperformed subgroup analysis conducted in the original meta-analyses.

\section{Estimation of summary effect}

We standardised the least active category as reference group across meta-analyses and then performed the meta-analysis of physical activity (ie, high vs low category) and each cancer site according to cancer indicators. We estimated summary effect measures and its 95\% CIs using random effect models.

\section{Heterogeneity between studies}

Heterogeneity between studies was estimated using the $\mathrm{I}^{2}$ measure of inconsistency ${ }^{3536}$ and $95 \%$ prediction intervals. The 95\% prediction interval accounts for heterogeneity between studies and represents the range in which a future study will lie. ${ }^{37}$

\section{Small study effect and excess significance biases}

Bias in the body of evidence was assessed by small study effect and excess significance tests. The presence of small study effects bias was assessed for each meta-analysis based on the regression asymmetry test proposed by Egger and colleagues. ${ }^{38}$ We considered small study effect bias when the Egger's test $\mathrm{P}$ value $<0.10$ and the magnitude of association in the largest study (smaller SE) of a meta-analysis was more conservative than the meta-analysis random effects estimate. ${ }^{39}$

The excess significance test was used to evaluate whether the expected number of studies (E) differs from the actual observed number of studies $(\mathrm{O})$ with statistically significant results $(\mathrm{P}<0.05)$ included in each meta-analysis, regardless of the direction of the association. ${ }^{40}$ The difference between $\mathrm{O}$ and $\mathrm{E}$ was evaluated using a two-sided binomial test. ${ }^{40}$ considering $\mathrm{P}<0.10$ for $\mathrm{O}$ greater than $\mathrm{E}$ (one-sided $\mathrm{P}<0.05$ ) as the statistical significance threshold.

All statistical analyses were performed using Stata V.13.0 (College Station, Texas, USA).

\section{Grading the evidence}

As proposed by previous umbrella reviews, ${ }^{26} 27303133$ we classified the evidence from meta-analysis with nominally statistically significant results $(\mathrm{P}<0.05)$ as strong, highly suggestive, suggestive or weak, following the criteria described in table 1 .

\section{Sensitivity analyses}

We performed credibility ceilings sensitivity analyses for associations showing at least weak evidence $(\mathrm{P}<0.05)$. Credibility ceilings evaluate potential spurious precision of the combined effect estimates. ${ }^{41}$ This tool re-estimates the meta-analysis pooled effect size using inflated variances for each study. The variance is 
Table 1 Summary of evidence grading for meta-analyses of cohort studies associating physical activity and risk of developing or dying from cancer

\begin{tabular}{|c|c|c|c|}
\hline \multirow[b]{2}{*}{ Evidence } & \multirow[b]{2}{*}{ Criteria used } & \multicolumn{2}{|l|}{ Decreased risk } \\
\hline & & $\begin{array}{l}\text { Any physical } \\
\text { activity }\end{array}$ & $\begin{array}{l}\text { Recreational } \\
\text { physical } \\
\text { activity }\end{array}$ \\
\hline Strong & $\begin{array}{l}{ }^{*} \mathrm{P}<10^{-6} ;>1000 \text { cases; } \mathrm{P}<0.05 \text { of } \\
\text { the largest study in a meta- } \\
\text { analysis; } \mathrm{I}^{2}<50 \% \text {; no small } \\
\text { study effectt; prediction interval } \\
\text { excludes the null value; no } \\
\text { excess significance bias } \neq\end{array}$ & None & $\begin{array}{l}\text { Colon cancer, inc. } \\
\text { or mort. }\end{array}$ \\
\hline $\begin{array}{l}\text { Highly } \\
\text { suggestive }\end{array}$ & $\begin{array}{l}{ }^{*} \mathrm{P}<10^{-6} ;>1000 \text { cases; } \mathrm{P}<0.05 \text { of } \\
\text { the largest study in a meta- } \\
\text { analysis }\end{array}$ & $\begin{array}{l}\text { Breast cancer, } \\
\text { inc. and all } \\
\text { cancer, mort. }\end{array}$ & None \\
\hline Suggestive & ${ }^{*} \mathrm{P}<10^{-3} ;>1000$ cases & $\begin{array}{l}\text { Colon cancer, inc. } \\
\text { or mort, lung inc. } \\
\text { and endometrial, } \\
\text { inc. }\end{array}$ & $\begin{array}{l}\text { All cancer, mort. } \\
\text { and lung, inc. }\end{array}$ \\
\hline Weak & ${ }^{*} P<0.05$ & $\begin{array}{l}\text { Meningioma, } \\
\text { inc. or mort, } \\
\text { multiple cancer } \\
\text { sites, inc. or mort, } \\
\text { pancreas, inc. }\end{array}$ & $\begin{array}{l}\text { Oesophageal, inc. } \\
\text { and meningioma, } \\
\text { inc. or mort. }\end{array}$ \\
\hline
\end{tabular}

${ }^{*} \mathrm{P}$ indicates the $\mathrm{P}$ values of the meta-analysis random effects model.

†Small study effect is based on the $P$ value from the Egger's regression asymmetry test $(P \leq 0.1)$ where the random effects summary estimate was larger compared with the point estimate of the largest study (smallest SE) in a meta-analysis.

$\neq$ Based on the $P$ value $(P>0.1)$ of the excess significance test using the largest study (smallest SE) in a meta-analysis as the plausible effect size.

inc., incidence; mort., mortality.

inflated by considering the number of studies showing effects on the same direction and the probability of each study for its true effect size to be in different direction from the one suggested by its point estimate. ${ }^{41}$ We evaluated a series of values to examine what credibility ceiling would be necessary to make the associations non-significant at the 0.05 level. ${ }^{41}$ Last, we performed a sensitivity analyses excluding each criterion used for grading the evidence to analyse the impact in the results.

\section{RESULTS}

\section{Description of meta-analyses}

Of the 2975 records retrieved from the search in databases, we finally selected a total of 19 systematic reviews of observational studies (figure 1). Systematic reviews of randomised controlled trials were eligible, but were not found in the literature search. Eleven out of 19 reviews (58\%) scored $\geq 6$ points in the 11-items AMSTAR criteria, indicating a moderate to high methodological quality (see online supplementary table S4). Limited statistical tests and sensitivity analyses were performed in original meta-analyses. Statistical significance was assessed in all reviews through the fixed/random effect 95\% CI (ie, none reported the exact $\mathrm{P}$ value). None of the reviews performed excess significance, credibility ceiling and $95 \%$ prediction intervals tests. $\mathrm{I}^{2}$ heterogeneity estimates $(\mathrm{n}=17,90 \%)$, small study effect test $(n=18,95 \%)$ and number of cases $(n=18,95 \%)$ were reported in the majority of the original meta-analyses (see online supplementary table S1).

Reviews included associations on 22 different cancer sites using 541 original studies, of which 297 (55\%) were cohort and $244(45 \%)$ case-control studies. Most of the original studies, 344 (64\%), had cancer incidence as outcome, 35 (6\%) mortality and $162(30 \%)$ incidence and mortality. A total of
725074 cancer cases and 42428 cancer deaths were included in these meta-analyses. All except for six comparisons (gastric mortality, oesophageal mortality, meningioma risk, kidney mortality, Hodgkin lymphoma risk, pancreatic mortality) included more than 1000 cases in the meta-analyses (see online supplementary tables S5 and S6).

Results presented below are based on the primary analysis ('any physical activity' and recreational physical activity approaches), which included only cohort studies.

\section{Summary effect size}

Eight out of the 26 meta-analyses(31\%) based on the any physical activity approach showed statistically significant associations $(\mathrm{P}<0.05)$ in the random effect model. From those cancer sites, summary random effect size showed a $0.93-0.71$ RR of cancer among those in the most active category as compared with the least active group. When the random effects $\mathrm{P}<10^{-6}$ was used as a threshold, only overall cancer mortality (RR $0.79 ; 95 \% \mathrm{CI}$ 0.75 to 0.85 ), colon cancer (RR $0.81 ; 95 \% \mathrm{CI} 0.75$ to 0.88 ) and breast cancer incidence (RR $0.87 ; 95 \% \mathrm{CI} 0.84$ to 0.90 ) remained statistically significant (figure 2 and see online supplementary table S7).

For recreational physical activity, 5 (25\%) out of 20 meta-analyses showed statistically significant associations based on the $\mathrm{P}<0.05$ threshold. Among those, only colon cancer (RR 0.79 ; 95\% CI 0.71 to 0.86 ) remained statistically significant at $\mathrm{P}<10^{-6}$ threshold (figure 3 and see online supplementary table $\mathrm{S} 8$ ).

\section{Heterogeneity between studies $-\mathrm{I}^{2}$ and $95 \%$ prediction intervals}

Half $(n=13)$ of the meta-analyses of the 'any physical activity' approach had $\mathrm{I}^{2}$ smaller than 25\%, whereas seven (27\%) (all-cancer mortality, colon, lung, gastric, bladder, multiple myeloma and ovary) showed moderate to high heterogeneity ( $\left.\mathrm{I}^{2} \geq 50 \%\right)$. Only all-cancer mortality and breast cancer presented a definite direction for the effect size ( $i e$, the intervals did not include the null value) when 95\% prediction intervals were considered (figure 2 and see online supplementary table S7).

Regarding recreational physical activity, seven out of 20 meta-analyses (35\%) had $\mathrm{I}^{2}>50 \%$ and 18 (90\%) included null value when $95 \%$ prediction intervals presented a definite direction for the effect size (figure 3 and see online supplementary table S8).

\section{Small study effects and excess significance biases}

Out of the 26 meta-analyses, four (15\%) (breast, all-cancer mortality, colon and pancreas) had a $\mathrm{P}<0.1$ on the Egger asymmetry test and the effect estimate of the largest study was more conservative compared with the summary random effects estimate, indicating potential small-study effect bias. Regarding the excess of significance bias, four (15\%) cancer sites (all-cancer mortality, colon, lung, gastric) had observed number of studies showing statistically significant results higher beyond chance than the expected (figure 2 and see online supplementary table S7).

For recreational physical activity, only multiple myeloma meta-analysis showed small-study effect bias, whereas only lung cancer presented evidence of excess significance bias (figure 3 and see online supplementary table S8).

\section{Robustness of evidence}

None of the associations were supported by strong evidence in the 'any physical activity' analyses. The associations between physical activity and breast cancer incidence and all-cancer 


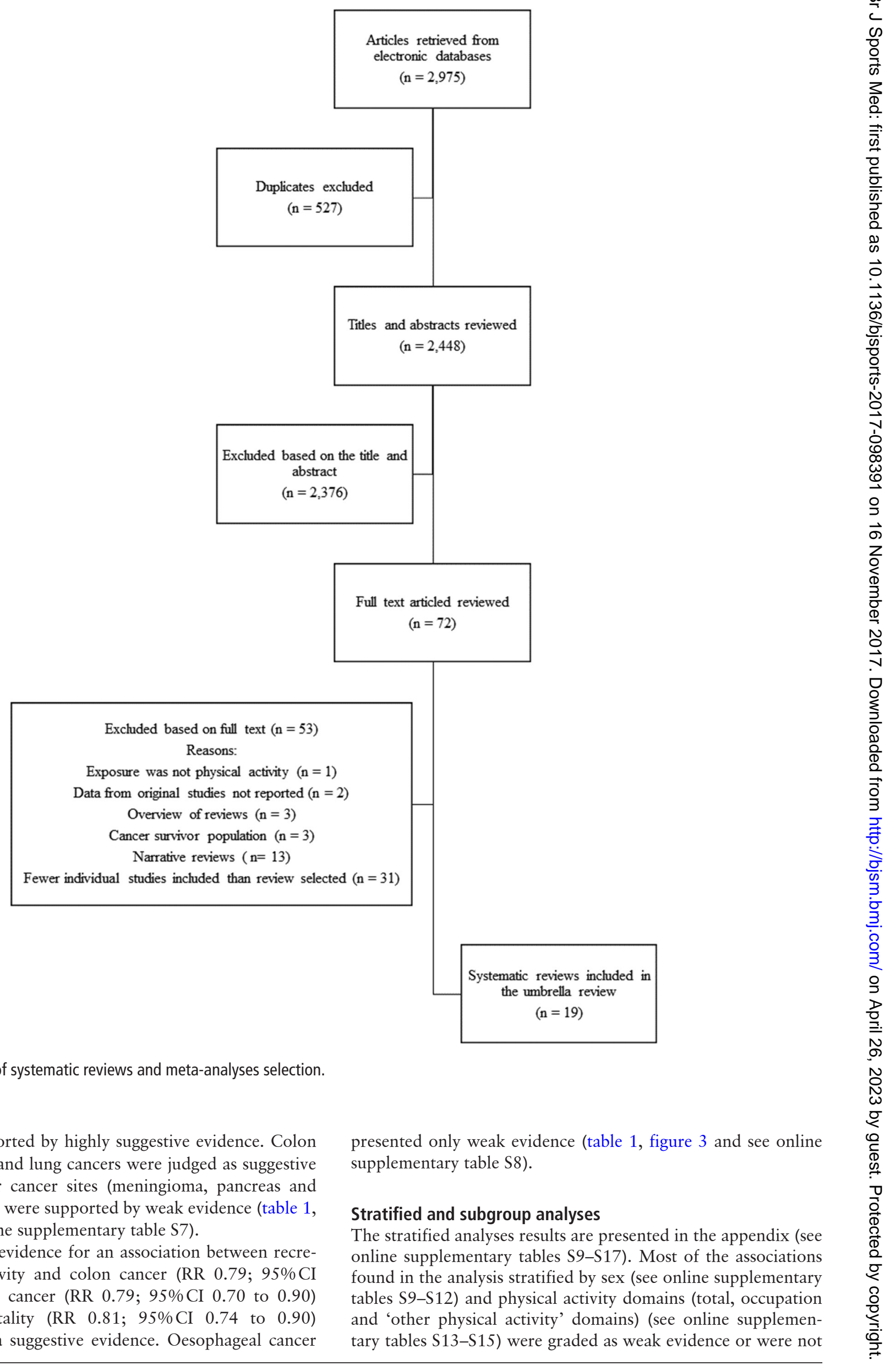

mortality were supported by highly suggestive evidence. Colon cancer, endometrial and lung cancers were judged as suggestive evidence. Two other cancer sites (meningioma, pancreas and multiple cancer sites) were supported by weak evidence (table 1 , figure 2 and see online supplementary table S7).

There was strong evidence for an association between recreational physical activity and colon cancer (RR 0.79; 95\% CI 0.71 to 0.86 ). Lung cancer (RR $0.79 ; 95 \%$ CI 0.70 to 0.90 ) and all-cancer mortality (RR $0.81 ; 95 \% \mathrm{CI} 0.74$ to 0.90 ) were supported by a suggestive evidence. Oesophageal cancer presented only weak evidence (table 1 , figure 3 and see online supplementary table S8).

\section{Stratified and subgroup analyses}

The stratified analyses results are presented in the appendix (see online supplementary tables S9-S17). Most of the associations found in the analysis stratified by sex (see online supplementary tables S9-S12) and physical activity domains (total, occupation tary tables S13-S15) were graded as weak evidence or were no 


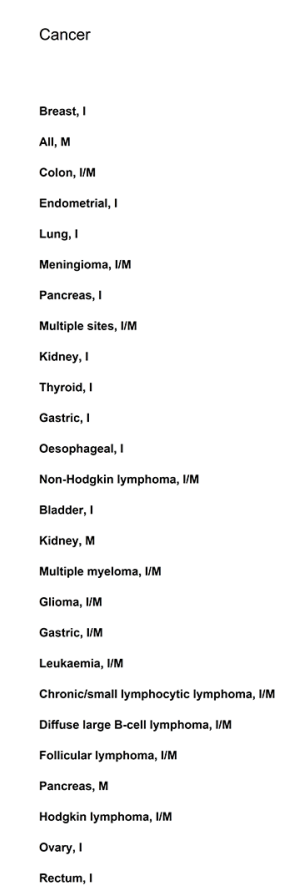

\begin{tabular}{|c|c|c|}
\hline $\begin{array}{c}\text { Number of } \\
\text { Studies }\end{array}$ & $\begin{array}{l}\text { Random } \\
\text { Effects P }\end{array}$ & $\begin{array}{c}\text { Number of } \\
\text { Cases }\end{array}$ \\
\hline 38 & $<10>-6$ & 116304 \\
\hline 25 & $<10 \wedge 6$ & 40469 \\
\hline 28 & $<10^{n-6}$ & 246123 \\
\hline 18 & $<10^{n-3}$ & 13583 \\
\hline 20 & $<10^{n-3}$ & 15777 \\
\hline 3 & $<0.05$ & 680 \\
\hline 19 & $<0.05$ & 7209 \\
\hline 11 & $<0.05$ & 16969 \\
\hline 9 & NS & 6035 \\
\hline 8 & NS & 1393 \\
\hline 6 & NS & 5295 \\
\hline 4 & NS & 896 \\
\hline 8 & NS & 6106 \\
\hline 11 & NS & 25174 \\
\hline 2 & NS & 69 \\
\hline 7 & NS & 1362 \\
\hline 3 & NS & 1194 \\
\hline 3 & NS & 2154 \\
\hline 6 & NS & 707 \\
\hline 4 & NS & 1125 \\
\hline 5 & NS & 1313 \\
\hline 5 & NS & 957 \\
\hline 5 & NS & 930 \\
\hline 3 & NS & 111 \\
\hline 9 & NS & 2467 \\
\hline 11 & NS & 33504 \\
\hline
\end{tabular}

Largest study No Small No Excess Evidence with $P<0.05$ study bias Sign. bias Grade

$$
\begin{aligned}
& + \\
& - \\
& + \\
& + \\
& + \\
& + \\
& + \\
& + \\
& + \\
& + \\
& + \\
& + \\
& + \\
& + \\
& + \\
& + \\
& +
\end{aligned}
$$

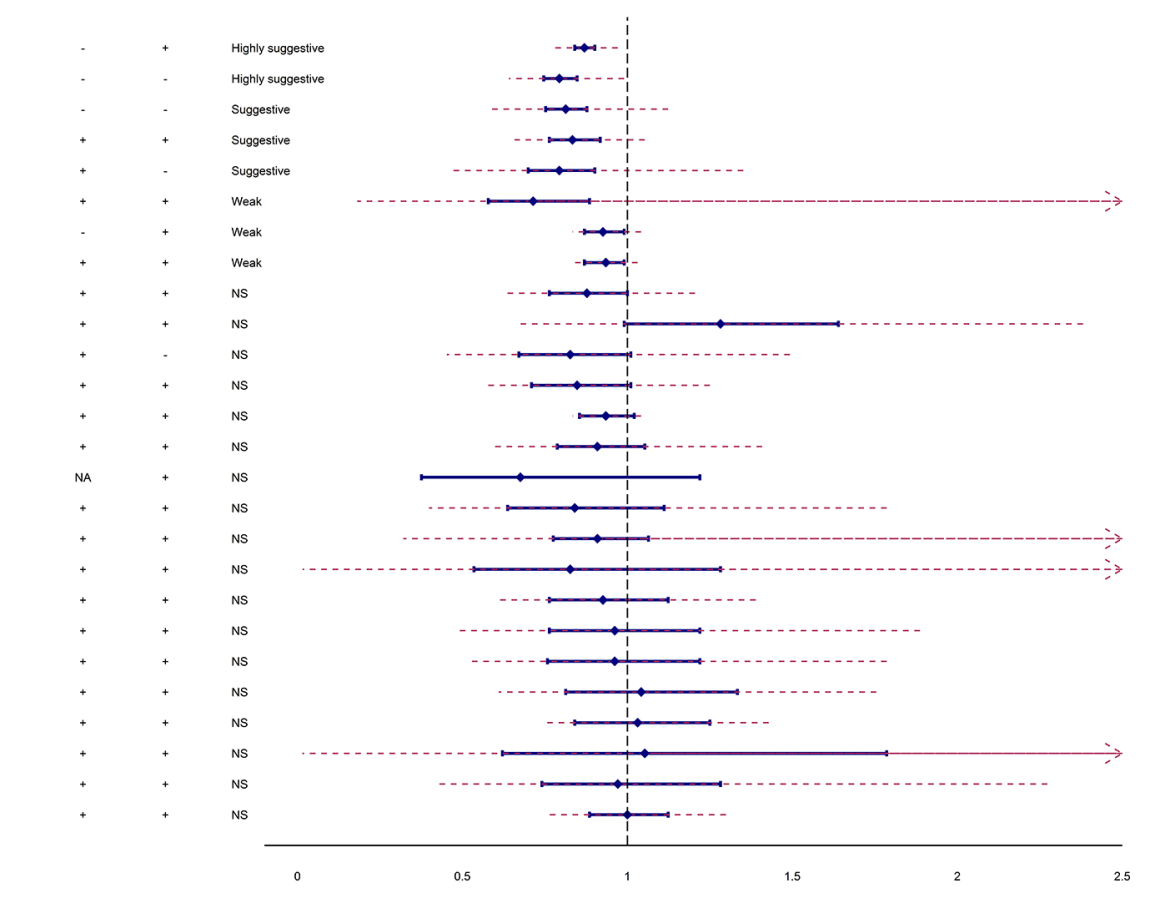

- - Prediction Interval _ Confidence Interval

Figure 2 Robustness of evidence grading for meta-analyses of cohort studies associating any physical activity and risk of developing or dying from cancer. Number of studies refers to number of studies included in the random effect model. Random effect $P$ refers to $P$ value of the summary random effects estimate. Number of cases refers to number of cancer cases or deaths included in the analysis. Largest study with $P<0.05$ refers to $P$ value of the largest study (smallest SE) in each meta-analysis. Small study bias is based on the $\mathrm{P}$ value from the Egger's regression asymmetry test $(\mathrm{P}<0.1)$ where the random effects summary estimate was larger compared to the point estimate of the largest study in a meta-analysis. Excess significance bias is based on the $P$ value $(P<0.1)$ of the excess significance test using the largest study (smallest $S E)$ in a meta-analysis as the plausible effect size. Evidence grading refers to robustness evidence grading criteria. I, incidence; $M$, mortality; I+M, incidence and mortality; NS, association not statistically significant $(P>0.05) ;+$, yes; - , no.

statistically significant, but data were generally more limited and many studies had not provided separate effects for men and women. Considering case-control studies, the association between any physical activity and colon and breast cancers were supported by highly suggestive evidence (see online supplementary table S16). For recreational physical activity, only breast cancer was supported by highly suggestive evidence in case-control studies (see online supplementary table S17).

The majority of the subgroup analyses conducted for each cancer were also supported by weak evidence or were not statistically significant. We found strong evidence for the associations between physical activity and distal colon cancer, breast cancer among women that never used hormone replacement therapy (HRT) (for estimates based on physical activity measured in hours/week and METs-hours/week, adjusted by adiposity), endometrial cancer (eg, for studies with number of cases higher than 3500 and with occupational physical activity measures) and non-cardia gastric cancer (see online supplementary tables S18-S39).

\section{Sensitivity analyses}

Of the eight meta-analyses showing random effects $\mathrm{P}<0.05$ in the 'any physical activity' approach, four (breast, colon, endometrial and all-cancer mortality) would remain statistically significant even with a $10 \%$ credibility ceiling and two (breast cancer and all-cancer mortality) even with a 20\% credibility ceiling (see online supplementary table S7 and figure S1). Two (colon cancer and all-cancer mortality) out of five meta-analyses of recreational physical activity showing random effects $\mathrm{P}<0.05$ remained statistically significant when $10 \%$ credibility was used (see online supplementary table S8 and figure S2), but none of those survived to $20 \%$ credibility ceiling.

When we performed sensitivity analyses excluding sequentially each criterion used in the grading of evidence, the association between any physical activity and breast cancer, stemming from cohort studies, was supported by strong evidence after excluding the small study effects criterion (see online supplementary table S40).

\section{DISCUSSION}

\section{Principal findings and possible explanations}

In this umbrella review summarising the evidence of associations between physical activity and different cancer sites, we synthesised data from 22 different cancer sites, 725074 cancer cases and 42428 cancer deaths and evaluated the credibility of the epidemiological evidence. In our primary analysis, stemming from cohort studies, physical activity showed a negative and statistically significant association $(\mathrm{P}<0.05)$ with seven cancer sites (colon, breast, endometrial, lung, oesophageal, pancreas and meningioma). However, we found that only the associations with colon cancer and breast cancer were supported by strong evidence and highly suggestive evidence, respectively.

The association between physical activity and incidence of breast and colon cancers was recognised long ago, ${ }^{12}$ whereas 


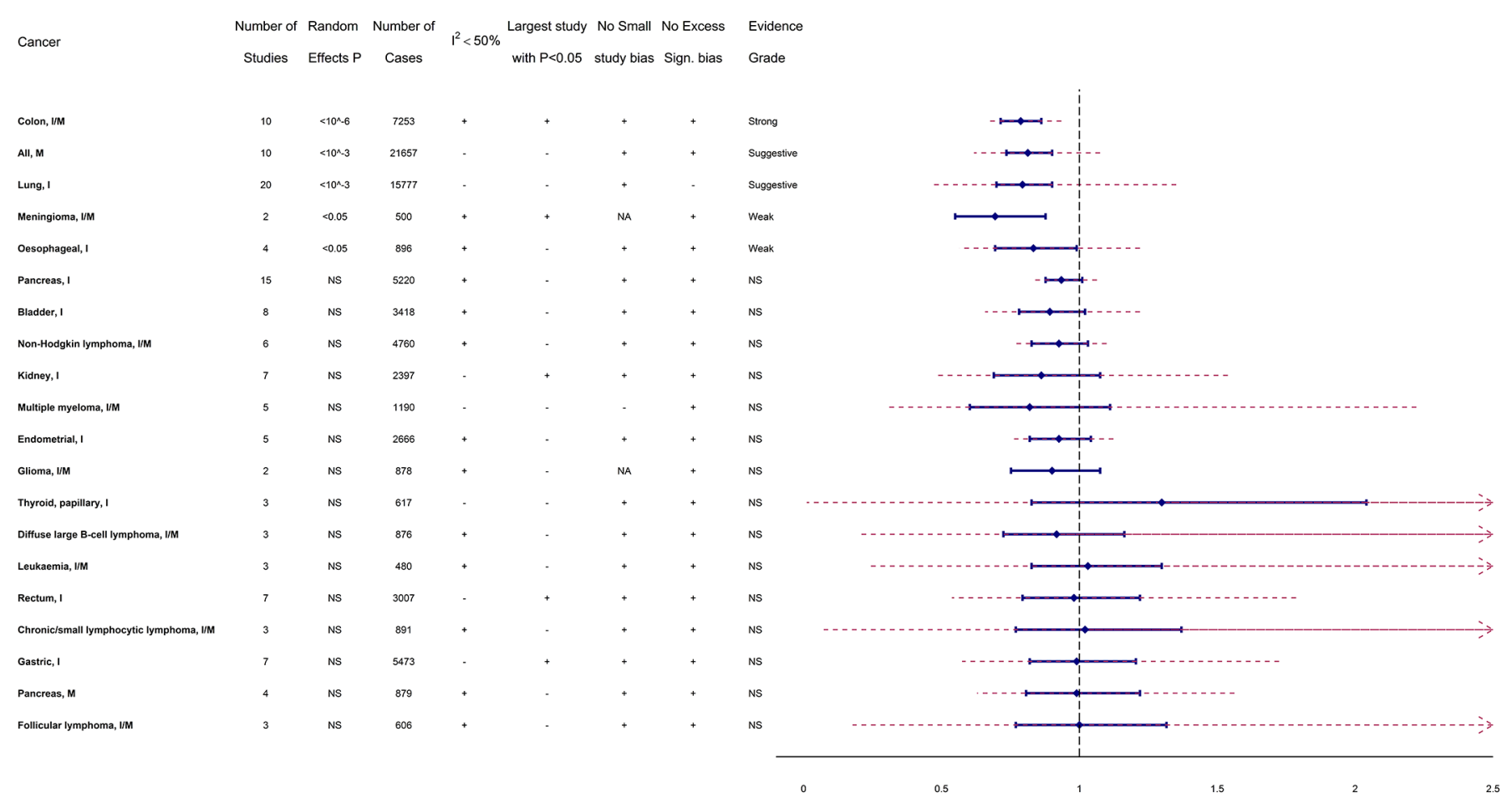

- - Prediction Interval — Confidence Interval

Figure 3 Robustness of evidence grading for meta-analyses of cohort studies associating recreational physical activity and risk of developing or dying from cancer. Number of studies refers to number of studies included in the random effect model. Random effect $P$ refers to $P$ value of the summary random effects estimate. Number of cases refers to number of cancer cases or deaths included in the analysis. Largest study with $\mathrm{P}<0.05$ refers to $P$ value of the largest study (smallest SE) in each meta-analysis. Small study bias is based on the $P$ value from the Egger's regression asymmetry test $(P<0.1)$ where the random effects summary estimate was larger compared to the point estimate of the largest study in a metaanalysis; Excess significance bias is based on the $\mathrm{P}$ value $(\mathrm{P}<0.1)$ of the excess significance test using the largest study (smallest $\mathrm{SE})$ in a metaanalysis as the plausible effect size. Evidence grading refers to robustness evidence grading criteria. I, incidence; $\mathrm{M}$, mortality; I+M, incidence and mortality; NS, association not statistically significant $(P>0.05) ;+$, yes; - , no.

associations with other cancer sites has emerged in the last decade. ${ }^{9-17}$ However, it is also plausible these associations may be flawed due to biases in the literature, overestimating the potential effect of physical activity on the incidence and mortality of cancer. In fact, this phenomenon has been as detected by previous umbrella reviews on other subjects. ${ }^{26} 27303133$

Through an array of statistical analyses, we found substantial uncertainty in the literature of physical activity and cancer. When stringent $\mathrm{P}$ value was considered $\left(\mathrm{P}<10^{-6}\right)$, only colon cancer, breast cancer and all-cancer mortality associations remained statistically significant. Moderate to high heterogeneity $\left(\mathrm{I}^{2} \geq 50 \%\right)$ was found in a third of the meta-analyses. When such heterogeneity was considered in the $95 \%$ prediction intervals, only colon cancer, breast cancer and all-cancer mortality presented a definite direction for the effect size. We identified few additional hints of bias in the literature though the small study effect and excess significance tests.

\section{Comparison with other studies}

Criteria for evaluating the evidence have been proposed by different researchers ${ }^{42-45}$ and organisations. ${ }^{12} 46$ The umbrella review approach evaluates the credibility of evidence using an array of statistical tests and sensitivity analyses to obtain hints of uncertainty and bias in the body of literature. Although it is not possible to estimate the exact extent or source of bias that affects the evidence on physical activity and cancer, the criteria that we use aim to capture indirectly the potential effect of biases.
The IARC and the WCRF have their own group of experts and criteria to judge the evidence of potential carcinogens to humans, which do not include detailed evaluation of bias in the body of literature. On the other hand, both organisations consider the biological mechanisms evidence, which is beyond the scope of this umbrella review.

Both IARC and WCRF found convincing evidence to support the association between physical activity and colon cancer, in agreement with the conclusions of our umbrella review for recreational physical activity. In the subgroup analysis, we found that only the association with distal colon cancer was supported by strong evidence, whereas proximal colon cancer evidence was judged as suggestive. Colon cancer had suggestive evidence for association with any physical activity with hints of heterogeneity and bias. There are plausible biological mechanisms supporting the association between physical activity and colon cancer, such as reducing body fatness, inflammation, insulin levels and insulin resistance. ${ }^{47}$

The IARC and WCRF classified the association between physical activity and breast cancer as sufficient (highest grade) and probable (second highest grade), respectively. We found highly suggestive evidence that physical activity decreases the risk of breast cancer, although we also observed small-study effect bias for this association. Small-study effect may either be related to bias or random error or heterogeneity. ${ }^{39}$ Regarding the heterogeneity, the WCRF, but not the IARC, provides separate grading of evidence for menopausal status, suggesting limited evidence for the association between moderate to vigorous physical activity 
and premenopausal breast cancer and convincing evidence for postmenopausal breast cancer. Similarly, we found that premenopausal and postmenopausal breast cancers were supported by weak and highly suggestive evidence, respectively. HRT is associated with increased risk of breast cancer and may be an important confounder in postmenopausal breast cancer studies. ${ }^{48}$ We found strong evidence supporting a negative association between physical activity and breast cancer among never HRT users (ie, analysis less prone to confounding). Additional biological evidence supporting the association between physical activity and breast cancer includes postulated effects on body fatness, certain hormone metabolisms (eg, fasting insulin, oestrogens and androgens) and strengthening of the immune system. ${ }^{48}$

Our findings are in agreement with IARC and WCRF, except for endometrial cancer, for which the association with physical activity is considered as probable evidence (second highest grade) by the WCRF. We found only suggestive evidence to support the association between any physical activity and endometrial cancer, because the most stringent $\mathrm{P}$ threshold $\left(\mathrm{P}<10^{-6}\right)$ and $95 \%$ prediction interval criteria were not satisfied. The $\mathrm{P}$ value criteria might be due to small number of cohort studies on endometrial cancer $(n=18)$ as compared with breast $(n=38)$ and colon cancer $(n=28)$ literature. In fact, when we considered both cohort and case-control designs, and therefore increased the statistical power, the association between physical activity and endometrial cancer was supported by highly suggestive evidence. However, case-control studies presented higher effect size estimates than cohort studies, probably reflecting more bias. The source of heterogeneity (ie, reflected in the $95 \%$ prediction intervals) in our results on endometrial cancer might be due to obesity status. Endometrial cancer is an obesity-related cancer, ${ }^{30}$ and body mass index (BMI) may mediate the association between physical activity and endometrial cancer. ${ }^{7}$ For instance, Moore et al found that leisure-time physical activity (LTPA) was associated with endometrial cancer only among individuals with high BMI. ${ }^{7}$ Other mechanisms suggested for this association are hormone-related, such as reducing insulin level and insulin resistance, decreasing estradiol and regulating oestrogen metabolism. ${ }^{49}$

Other cancer sites (oesophageal, meningioma, lung, pancreas) showed less consistent results with substantial uncertainty in the literature. None of these cancer sites supported more stringent $\mathrm{P}$ thresholds used to avoid spurious precision results derived from meta-analyses of observational studies. Three (oesophageal, meningioma, pancreas) out of four did not have more than 1000 cases to state conclusion. Heterogeneity ( $\mathrm{I}^{2}$ and $95 \%$ prediction intervals) and/or bias (small-study effect and excess significant biases) were present in all these meta-analyses. Finally, other cancer sites (bladder, chronic/small lymphocytic lymphoma, diffuse large B-cell lymphoma, follicular lymphoma, gastric, glioma, Hodgkin and non-Hodgkin's lymphoma, kidney, leukaemia, multiple myeloma, ovary, rectum, thyroid) did not show statistically significant associations at $\mathrm{P}<0.05$ threshold. IARC and WCRF also have also considered these associations between physical activity and these cancer sites with limited evidence. However, a recent pooled data from 12 cohorts examined the association between LTPA and 26 types of cancer and 187000 cancer cases. LTPA was inversely associated (at $\mathrm{P}<0.05$ ) with 13 cancer sites (colon, breast, endometrial, oesophageal adenocarcinoma, liver, kidney, gastric cardia, myeloid leukaemia, myeloma, head and neck, rectal and bladder) and positively associated with melanoma and prostate cancer. ${ }^{7}$ Although this paper was not included in our umbrella review, as did not meet the eligibility criteria (ie, was not a systematic review of literature), we recognise its importance to the physical activity and cancer literature. Thus, we applied our grading evidence criteria to their
What is already known?

- Physical activity has been traditionally linked with lower risks of breast and colon cancer.

- Recently, multiple meta-analyses have showed that physical activity might be additionally associated with lower risk of several other cancer sites.

- If these associations are causal, a substantial burden of cancer could be avoided worldwide, but it is also plausible that some associations may be flawed due to biases in the literature.

What are the new findings?

- This umbrella review synthesises and evaluates the robustness of evidence and appraises uncertainty and bias in the body of literature of the association between physical activity and the risk of developing or dying from cancer.

- Of the 22 major anatomical cancer sites included in our primary analysis, only colon cancer and breast cancer were supported by strong or highly suggestive evidence.

- Evidence from other cancer sites was less consistent, presenting hints of uncertainty and bias in the literature.

findings. We found that only the association between LTPA and breast, kidney, melanoma and lung cancers were supported by strong evidence (see online supplementary table S41). Therefore, despite the uncertainty or bias that was found for many malignancies, these associations could be confirmed as genuine in the future.

Physical activity is associated with obesity and diabetes and these phenotypes have also been associated to cancer at several sites. $^{265051}$ Deciphering the exact causal contribution of each of these factors is not easy. Both low within-population variability of and measurement error for physical activity are also important concerns. Future prospective cohort studies with objective measures (eg, through accelerometers) of physical activity trajectories may reduce misclassification and, therefore, reduce current uncertain evidence for some cancer sites. In parallel, randomised controlled trials of physical activity may help address directly the causal effects. Given the very long follow-up required to study cancer outcomes, these studies are difficult to conduct. However, given its potential major importance, physical activity interventions may need to be studied with large randomised trials, much like other interventions (eg, drugs) have been studied. ${ }^{5253}$

\section{Limitations}

Umbrella reviews rely on methodological quality and report transparency of meta-analyses. Despite the fact that the systematic reviews included in our umbrella were of moderate to high methodological quality and were published on average in 2014 (2005-2017), some studies may have not been included either because systematic reviews did not identify them or they were too recent to be included. ${ }^{7}$ We reperformed most of the analysis reported in systematic reviews; however, substantial data were missing from some subgroup analyses (see online supplementary table S42-S46). We encourage future systematic reviews to report each individual-study estimate included in its primary and main subgroup (sex, cancer location, histology) meta-analyses. 
Our analyses are based on the comparison between most active and least active groups. A limitation of this approach is that measurement and classification of physical activity across studies are heterogeneous and might not be comparable. Finally, tests used to obtain hints of bias in the body of evidence (smallstudy effect and excess significance tests) have low power if the meta-analyses include less than 10 studies and they may not identify the exact source of bias. ${ }^{3954}$

\section{CONCLUSION}

Despite the fact that physical activity has been associated with a lower risk of several cancers in the literature, the associations for only colon cancer and breast cancer were supported by strong or highly suggestive evidence, respectively. Evidence from other cancer sites was less consistent, presenting hints of uncertainty and/or bias, but could be confirmed as genuine in the future.

\section{Author affiliations}

Departamento de Medicina Preventiva, Faculdade de Medicina FMUSP, Universidade de São Paulo, São Paulo, Brazil

${ }^{2}$ Núcleo de Pesquisas Epidemiológicas em Nutrição e Saúde, Universidade de São Paulo, São Paulo, Brazil

${ }^{3}$ Department of Hygiene and Epidemiology, University of Ioannina School of Medicine, Ioannina, Greece

${ }^{4}$ Prevention Research Collaboration, Sydney School of Public Health, University of Sydney, Sydney, Australia

${ }^{5}$ Harvard Medical School, Brigham and Women's Hospital, Boston, Massachusetts, USA

${ }^{6}$ Department of Epidemiology, Harvard TH Chan School of Public Health, Boston, Massachusetts, USA

${ }^{7}$ Department of Epidemiology and Biostatistics, School of Public Health, Imperial College London, London, London, UK

${ }^{8}$ Stanford Prevention Research Center, Department of Medicine, Stanford University School of Medicine, Stanford, California, USA

${ }^{9}$ Department of Health Research and Policy, Stanford University School of Medicine, Stanford, California, USA

${ }^{10}$ Department of Biomedical Data Science, Stanford University School of Medicine, Stanford, California, USA

${ }^{11}$ Department of Statistics, Stanford University School of Humanities and Sciences, Stanford, California, USA

Contributors All authors conceived and designed the study. LFMR, JPRL and THS acquired and collated the data. LFMR and GM analysed the data. All authors drafted and critically revised the manuscript for important intellectual content and gave final approval of the version to be published.

Funding This work was supported by São Paulo Research Foundation (FAPESP), grant\#2014/25614-4 (funding was obtained from FAPESP as part of LFMR doctoral scholarship); World Cancer Research Fund International Regular Grant Programme (2014/1180 to KKT; funding was obtained from World Cancer Research Fund (WCRF UK), as part of the World Cancer Research Fund International grant programme). JPRL is postdoctoral fellowship from the University of Sydney (Australia) (Project Code: U2334).

\section{Competing interests None declared.}

Provenance and peer review Commissioned; externally peer reviewed.

(c) Article author(s) (or their employer(s) unless otherwise stated in the text of the article) 2018. All rights reserved. No commercial use is permitted unless otherwise expressly granted.

\section{REFERENCES}

1 World Cancer Research Fund American Institute for Cancer Research. Food, nutrition, physical activity, and the prevention of cancer:a global perspective. Washington, DC: American Institute for Cancer Research, 2007

2 International Agency for Research on Cancer. IARC handbook of cancer prevention. Weight control and physical activity. 6. IARC press: Lyon, 2002.

3 Pizot C, Boniol M, Mullie P, et al. Physical activity, hormone replacement therapy and breast cancer risk: A meta-analysis of prospective studies. Eur J Cancer 2016;52:138-54.

4 Neilson HK, Farris MS, Stone CR, et al. Moderate-vigorous recreational physical activity and breast cancer risk, stratified by menopause status: a systematic review and meta-analysis. Menopause 2017;24:322-44.

5 Wolin KY, Yan Y, Colditz GA, et al. Physical activity and colon cancer prevention: a meta-analysis. Br J Cancer 2009;100:611-6.
6 Boyle T, Keegel T, Bull F, et al. Physical activity and risks of proximal and distal colon cancers: a systematic review and meta-analysis. J Natl Cancer Inst 2012;104:1548-61.

7 Moore SC, Lee IM, Weiderpass E, et al. Association of leisure-time physical activity with risk of 26 types of cancer in 1.44 million adults. JAMA Intern Med 2016;176:816-25.

8 Keum N, Bao Y, Smith-Warner SA, et al. Association of physical activity by type and intensity with digestive system cancer risk. JAMA Oncol 2016:2:1146-53.

9 Keimling M, Behrens G, Schmid D, et al. The association between physical activity and bladder cancer: systematic review and meta-analysis. Br J Cancer 2014;110:1862-70.

10 Schmid D, Behrens $G$, Keimling M, et al. A systematic review and meta-analysis of physical activity and endometrial cancer risk. Eur J Epidemiol 2015;30:397-412.

11 Behrens G, Jochem C, Keimling M, et al. The association between physical activity and gastroesophageal cancer: systematic review and meta-analysis. Eur J Epidemiol 2014;29:151-70.

12 Psaltopoulou T, Ntanasis-Stathopoulos I, Tzanninis IG, et al. Physical activity and gastric cancer risk: systematic review and meta-analysis. Clin I Sport Med 2016;26:445-64.

13 Niedermaier T, Behrens G, Schmid D, et al. Body mass index, physical activity, and risk of adult meningioma and glioma: A meta-analysis. Neurology 2015;85:1342-50.

14 Behrens G, Leitzmann MF. The association between physical activity and renal cancer: systematic review and meta-analysis. Br J Cancer 2013;108:798-811.

15 Brenner DR, Yannitsos DH, Farris MS, et al. Leisure-time physical activity and lung cancer risk: a systematic review and meta-analysis. Lung Cancer 2016;95:17-27.

16 Zhong S, Chen L, Lv M, et al. Nonoccupational physical activity and risk of ovarian cancer: a meta-analysis. Tumour Biol 2014;35:11065-73.

17 Behrens G, Jochem C, Schmid D, et al. Physical activity and risk of pancreatic cancer: a systematic review and meta-analysis. Eur J Epidemiol 2015;30:279-98.

18 Liu Y, Hu F, Li D, et al. Does physical activity reduce the risk of prostate cancer? a systematic review and meta-analysis. Eur Urol 2011;60:1029-44.

19 Hallal PC, Andersen LB, Bull FC, et al. Global physical activity levels: surveillance progress, pitfalls, and prospects. Lancet 2012;380:247-57.

20 Dwan K, Gamble C, Williamson PR, et al. Systematic review of the empirical evidence of study publication bias and outcome reporting bias - an updated review. PLOS One 2013:8:e66844.

21 Young NS, loannidis JP, Al-Ubaydli O. Why current publication practices may distort science. PLoS Med 2008;5:e201.

22 loannidis JP. Why most published research findings are false. PLoS Med 2005;2:e124.

23 Taubes G. Epidemiology faces its limits. Science 1995;269:164-9.

24 Boffetta P, McLaughlin JK, La Vecchia C, et al. False-positive results in cancer epidemiology: a plea for epistemological modesty. J Nat/ Cancer Inst 2008; 100:988-95.

25 Tsilidis KK, Papatheodorou SI, Evangelou E, et al. Evaluation of excess statistical significance in meta-analyses of 98 biomarker associations with cancer risk. J Nat/ Cancer Inst 2012;104:1867-78.

26 Tsilidis KK, Kasimis JC, Lopez DS, et al. Type 2 diabetes and cancer: umbrella review of meta-analyses of observational studies. BMJ 2015;350:g7607.

27 Ioannidis JP, Zhou Y, Chang CQ, et al. Potential increased risk of cancer from commonly used medications: an umbrella review of meta-analyses. Ann Oncol 2014;25:16-23.

28 Schoenfeld JD, loannidis JP. Is everything we eat associated with cancer? a systematic cookbook review. Am J Clin Nutr 2013;97:127-34.

29 Kyzas PA, Denaxa-Kyza D, loannidis JP. Almost all articles on cancer prognostic markers report statistically significant results. Eur J Cancer 2007;43:2559-79.

30 Kyrgiou M, Kalliala I, Markozannes G, et al. Adiposity and cancer at major anatomical sites: umbrella review of the literature. BMJ 2017;356:j477.

31 Markozannes G, Tzoulaki I, Karli D, et al. Diet, body size, physical activity and risk of prostate cancer: An umbrella review of the evidence. Eur J Cancer 2016;69:61-9.

32 World Cancer Research Fund International. Continuous update project findings \& reports. 2017 http://www.wcrforg/int/research-we-fund/continuous-update-projectfindings-reports (accessed $03 \mathrm{Apr}$ 2017).

33 Bellou V, Belbasis L, Tzoulaki I, et al. Systematic evaluation of the associations between environmental risk factors and dementia: An umbrella review of systematic reviews and meta-analyses. Alzheimers Dement 2017;13:406-18.

34 Shea BJ, Grimshaw JM, Wells GA, et al. Development of AMSTAR: a measurement too to assess the methodological quality of systematic reviews. BMC Med Res Methodol 2007;7:10.

35 Hardy RJ, Thompson SG. Detecting and describing heterogeneity in meta-analysis. Stat Med 1998;17:841-56.

36 Ioannidis JP, Patsopoulos NA, Evangelou E. Uncertainty in heterogeneity estimates in meta-analyses. BMJ 2007;335:914-6.

37 Riley RD, Higgins JP, Deeks JJ. Interpretation of random effects meta-analyses. BMJ 2011;342:d549.

38 Egger M, Davey Smith G, Schneider M, et al. Bias in meta-analysis detected by a simple, graphical test. BMJ 1997;315:629-34.

39 Sterne JA, Sutton AJ, loannidis JP, et al. Recommendations for examining and interpreting funnel plot asymmetry in meta-analyses of randomised controlled trials. BMJ 2011;343:d4002. 
40 Ioannidis JP, Trikalinos TA. An exploratory test for an excess of significant findings. Clin Trials 2007:4:245-53.

41 Salanti G, Ioannidis JP. Synthesis of observational studies should consider credibility ceilings. J Clin Epidemiol 2009;62:115-22.

42 Hill AB. The environment and disease: Association or causation? Proc R Soc Med 1965:58:295-300.

43 Guyatt GH, Oxman AD, Vist GE, et al. GRADE: an emerging consensus on rating quality of evidence and strength of recommendations. BMJ 2008:336:924-6.

44 loannidis JP. Exposure-wide epidemiology: revisiting Bradford Hill. Stat Med 2016:35:1749-62

45 Lawlor DA, Tilling K, Davey Smith G. Triangulation in aetiological epidemiology. Int J Epidemiol 2016;45:1866-86.

46 USDepartment of Health Education and Welfare. Smoking and health. Report of the Advisory Committee to the Surgeon General of the Public Health Service Publication 1103. Washington,DC: US Government Printing Office, 1964.

47 World Cancer Research Fund/American Institute for Cancer Researh. Continuous update project report. Food, nutrition, physical activity, and the prevention of colorectal cancer, 2011.
48 World Cancer Research Fund/American Institute for Cancer Resarch. Continuous update project report. Continuous update project report. Food, nutrition, physical activity, and the prevention of breast cancer, 2010.

49 World Cancer Research Fund/American Institute for Cancer Research. Continuous update project report. Food, nutrition, physical activity, and the prevention of endometrial cancer, 2013.

50 Lauby-Secretan B, Scoccianti C, Loomis D, et al. Body fatness and cancer-viewpoint of the IARC working group. N Engl J Med 2016;375:794-8.

51 Giovannucci E, Harlan DM, Archer MC, et al. Diabetes and cancer: a consensus report. Diabetes Care 2010;33:1674-85.

52 Naci H, loannidis JP. Comparative effectiveness of exercise and drug interventions on mortality outcomes: metaepidemiological study. BMJ 2013;347:f5577.

53 Dal-Ré R, Bracken MB, loannidis JP. Call to improve transparency of trials of nonregulated interventions. BMJ 2015;350:h1323.

54 Lau J, loannidis JP, Terrin N, et al. The case of the misleading funnel plot. BMJ 2006;333:597-600. 